\title{
Refreshing Quantification and other Ploys to Give Up the Habit
}

\section{A Repertoire of Relations, Identities, and Rhetorical Devices in Smoking Cessation Applications}

\author{
Ștefania Matei ${ }^{1}$, Cosima Rughiniș ${ }^{1}$, and Răzvan Rughiniș ${ }^{2}$ \\ ${ }^{1}$ University of Bucharest, Department of Sociology, Bucharest, Romania \\ \{stefania.matei, cosima.rughinis\} asas.unibuc.ro \\ ${ }^{2}$ University Politehnica of Bucharest, Department of Computer Science, \\ Splaiul Independenței 313, Bucharest, Romania \\ razvan.rughinis@cs.pub.ro
}

\begin{abstract}
In this paper we analyze 26 smoking cessation applications on Android OS focusing on how they address their implied users. We identify 'refreshing quantification' as a main method, which endorses a portrait of the users as myopic in risk perception, but heroic in their individual pursuit to reach the non-smoker identity. App-created relationships and identities give rise to a temporal order based on contemplating the past and anticipating the future. Users are guided towards an autonomy-centered identity project, which renders them accountable for success or failure in smoking cessation. Users' experience of smoking cessation is co-constructed in their interaction with the app-coach and with peers. Apps and peers offer diagnoses, advice, labels that populate the world in which the would-be ex-smokers pursue their project.
\end{abstract}

Keywords: Smoking Cessation, Implied User, Android Applications, Refreshing Quantification, Rhetorical Device.

\section{Introduction}

Applications designed to assist efforts at giving up smoking have become increasingly complex and diverse. They are neither strictly a manual of self-government with motivational claims directed towards renouncing a habit, nor a simple record of a behavior. Instead, they provide an identity project with moral implications through which users are transformed from the position of irresponsible consumers of cigarettes to the position of responsible, well-documented and self-controlled persons. We aim to examine this process, in light of frequent skepticism expressed about the effectiveness of behavioral change apps.

Previous research on smoking cessation programs has looked into intervention designs, short- and long-term impact, and users' experiences. Impact evaluation metaanalyses indicate that Internet interventions seem promising for long-term (6 month) results, especially if they are interactive and tailored to the individual user [1]. Mobile 
text messaging interventions also seem to be effective in supporting long term cessation [2]. Still, mobile applications for smoking cessation have not yet been systematically evaluated as regards impact. Anyway, a review of 47 iPhone apps indicates that they have a low degree of conformity with clinical practice guidelines [3]; the best followed guideline refers to offering rewards and presenting the benefits of quitting, while least attended guidelines refer to recommending approved medication and counselling. Apps are often developed independently of medical expertise, thus raising the question: what are the persuasive approaches put in place to support users through a difficult personal transformation?

\section{Structural Design and Implied Users}

In our inquiry we start from the concept of the 'implied user' - a productive conceptual instrument in analyzing human-device interaction throughout the life of an application: starting from its creation and design and ending up with its circulation and utilization.

Either approached as a rhetorical tool for designers (a "scenic feature of the design space" [4]) or as a human prototype that is inscribed into persons through interaction with application scripts [5], the 'user' has received much scientific attention, particularly in the study of software.

In this thread of research, the 'implied user' is not assembled as a statistical or psychological aggregate of real people who use applications. In some cases it is retrieved as an assumed model of person, formulated through discourse in interaction, which designers and other participants in software development require in order to make sense of the situation, coordinate their actions, and account for their decisions [4-9]. From this perspective, users' expected behaviors are interweaved with the product development requirements [6] [7] [8].

Secondly, in other types of research, the 'implied user' is a typification embedded in the structural design of the application [9] which can be retrieved by examining the parameters, settings, functions and possible modes of operating an application, without reference to how it was designed or actually used. Empirical studies based on this perspective analyzed software programs as modes of 'performing users' by imputing them certain qualities [10].

Thirdly, in a related understanding, the 'user' does not take the form of a repository of needs and interests, but it represents a mode of behavior and habits acquired through and by using an application. In this sense, the 'user' might be addressed as an outcome of engaging with technology, therefore being a projection of the influence that an application might have in real-life situations. Even if this approach allows for the study of users on different levels, empirical research is focused mainly on evaluating and measuring the impact of software programs on human behavior [11].

Last but not least, investigations of actual use can contribute to highlight discrepancies between the implied users' and the flesh-and-bones users' forms of interaction [12]. 
In this conceptual landscape, we start from the perspective that the 'user' is part of a system of categories useful for organizing specific situations that appear in relation to a software application. Even if the 'user' is often assimilated with a consumer, customer, or operator, thus receiving a factual and observable form of existence, it is essentially a rhetorical construction that gains meaning through practices in which descriptions of human actions are made to be relevant in accounts that guide understanding.

In this paper we analyze 26 smoking cessation applications on Android OS (presented in Fig. 1 in the descending order of their popularity), and we inquire into their repertoire of proposed relationships, user identities, and rhetorical styles. We focus on how the implied user is configured through communication invited by the apps. The increasing diversity of the app environment should benefit from a systematic examination, offering developers information on current and possible ways of approaching users.

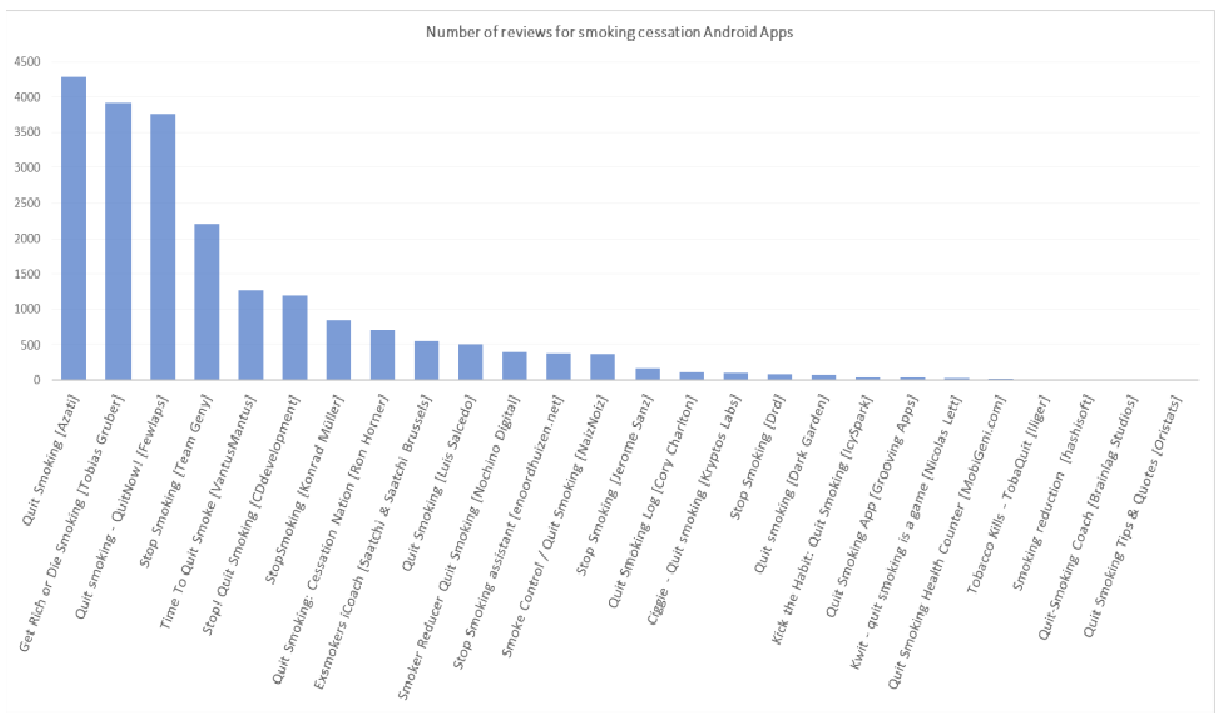

Fig. 1. Android apps for smoking cessation sorted by number of user reviews

Many mobile applications visible when searching Google Play for "quit smoking" share important features when formulating and approaching the problem they profess to solve. Users can launch the application by deciding to quit smoking: the planned date of renouncing cigarettes is inputted as the main application propellant. Users may also introduce other details about their past smoking behavior. From that moment a counter is enabled, measuring the time passed without smoking, and displaying statistics in accordance with input data. Former smokers are motivated to avoid relapse by accumulating achievements in proportion to the period spent without smoking. These digital rewards consist in the display of health benefits, in consumption proposals based on money savings, or other motivators. The applications offer various incentives: tips and tricks, gamified environments, surveys, questions of reflection, 
opportunities of interacting with peers, the possibility to maintain a dialogue with the application etc.

We anticipate a general conclusion: the implied user of the examined applications is a lone rider, unassisted by medicine or counseling, solely equipped with a mobile device that works as a time traveling machine. The user is engaged in an initiatory journey having to pass from a dangerous to a safe world with only one imperative: do not smoke. The mobile is an omniscient companion which gives advice and other super-natural powers that help in fighting the pitfalls encountered in the mad world of cigarettes. Cessation is framed as time travel, in which users can reach almost instantaneously each part of their life by contemplating the must-get-rid-of-it past and by envisaging a promising future. In this sense, the main application operations function as a myopia-correcting gear by bringing about a quantitative makeup of the world as a frame of reference, which may also be shared with other users, on local forums or social media. Users are addressed as knowledge seekers afflicted by short-sightedness and misperception of risks. The 'refreshing quantification' modules make visible for users those risks and benefits that they, presumably, could not see. Applications approach these cognitive limitations by transforming general and prevalent risks in individual self-inflicted threats. We argue that this dominant implied user is problematic in light of the high frequency of relapse among smokers who start to quit.

\section{Relationships in the App World}

Smoking cessation applications involve the users in four main types of mediated relationships, embedded in the architecture, technical features, and possibilities of engagement they propose. These relationships create links between various components of the process of quitting and represent the structural ground on which the personal transformation path develops.

Firstly, the applications propose a relationship with oneself, created through increased self-monitoring procedures. Apps often include a 'refreshing quantification' module that offers numerical estimates of current health risks and their future evolution, continuously updated. Users are informed about the micro-level transformations of their bodies, and the macro-level changes that will materialize in the next days, months, and years, with a rhetoric of high precision. Apps thus offer a 'microscope' and a 'telescope' to examine one's present and future body (Fig. 2a). Of course, all these numbers are not personalized estimates related to the current state of the users' body, but aggregate guesstimates that input users' succinct smoking profile into formulas relying on publicly available statistics of risks computed on large populations.

Each of the examined applications attempts to render smoking behavior visible, by requesting users to describe it through numbers in a smoking profile. Applications use quantification to objectify smoking by transposing it in something easily measurable (e.g. number of cigarettes smoked per day, number of cigarettes in a pack), and to highlight the relevance of cost (e.g. cost per pack of cigarettes). Beside these, there are other indicators that shape the awareness of smoking behavior from different points of view (e.g. time spent per cigarette, which is framed as a cost). All these 
indicators are used to define the position from which users are apprehended, to establish what counts in their transformation, and how it should be counted.

This first stage of quantification involves initial data input and is mandatory. Thus users are requested to think about their past smoking behavior and to objectify it in numbers. This assessment makes smoking appear uniform in time, which is not always the case. By continuing to use the application, the former smokers accept that this type of conventional quantification (number of cigarettes per day, price per pack etc.) is meaningful for describing and managing their behavior.

Moreover, by producing numerical estimates, users implicitly consent with (and legitimate) the quantitative language and representations of their worlds and actions. On the one hand, estimates provided by the application depend on the inputs offered by the user, and thus the plausibility of application metrics is a direct function of the credibility of users' initial information. On the other hand, there is also a symbolic connection: through agreeing to provide succinct generalizations of one's past behavior, users also agree to accept broad generalizations of their future. In this way, an implicit contract is established between users and smoking cessation applications, through a common and agreed upon quantitative language of self-description.

Secondly, the applications develop users' relationships with a gameplay environment. Applications may frame the quitting process as a game or offer minigames either as future rewards or as means to distract attention from craving. For example, they include in the quantification module sets of game-like achievements, which users unlock after being smoke free for a certain time (Fig. 2b).

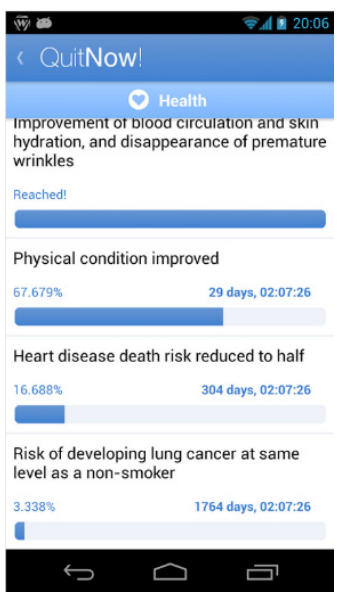

a) 'QuitNow': Present and future

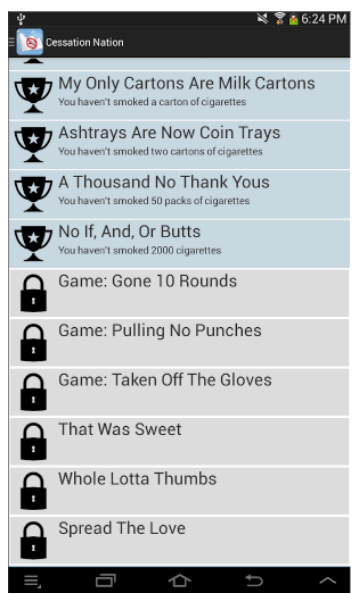

b) 'Cessation Nation': Gamification

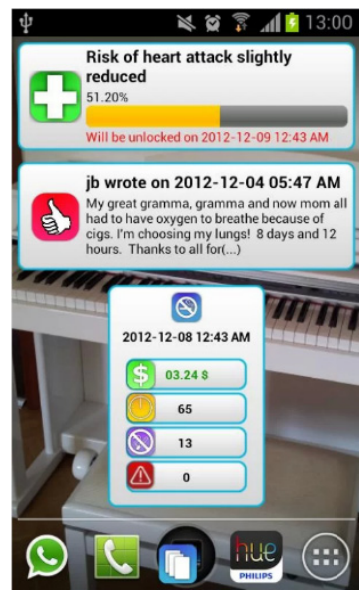

c) 'Stop! Quit Smoking': Forum talk

Fig. 2. Types of relationships: with self, the gameplay environment, and peers

The achievements function as evidence-based credentials [13] which account in communicating merits [14] and establishing relevant targets [15]. By using achievements, the applications provide a vocabulary of relevance as a descriptor of users' behavior . Thus they create a shared space in which a sense of purpose is continuously maintained through an infrastructure of sustained commitment. 
Gameplay elements, understood as a productive media to support interaction between various actors [16], introduce users in a meaningful story developed in parallel with their embodied experience. This is a story of courageous transformation, in which users are the main protagonists in search for the ultimate "powers of nonsmoking". In strongly gamified applications persistent smoking cessation is attested by a first class cup, a paramount medal, or a final congratulation message, all of them certifying the end of the difficult pursuit. As in a usual game, users are informed that they can try again and again to pass an obstacle, but through effort and self-training the success is guaranteed. By highlighting the 'ultimate achievement' in the interaction with users, a sense of purpose is integrated in an ongoing process of difficult, often painful transformation. A couple of applications also make visible the level of difficulty proposed to users: some periods or tasks are framed as being more difficult than others.

Another type of mediated relationships refers to relationships with a community of peers. Applications can host their own forums (Fig. 2c) which enable users to share their experiences, progress, and achievements. They can also post on Facebook or other social media. Applications integrate various forms of sociability in their design, by creating a platform of dialogue and support and by bringing together participants willing to share their experience with others. These online communities grow in parallel with other applications features, creating a "new application within the main application" which functions on its own rationale. Online communities can offer different styles of communication, advice, and frames from the dominant app logic. Users get the opportunity to talk about their experiences, co-constructing them with the app and peers.

Last, but not the least, applications create users' relationship with a quitting couch. Apps offer tips (Fig. 3a), either on request or as a static page. They can also attempt to delay and prevent users' smoking (Fig. 3b) by addressing their desire to smoke through the interaction with a personal assistant made visible on the mobile screen.

Smoking-cessation applications propose to function as an assistive technology for a kind of short-sightedness: they provide smokers the perceptive tools for apprehending the temporal structure of the world, in order to successfully adhere to their cessation plan. To this purpose, they use extensive procedure of framing smoking, risks and benefits, bringing about the well-documented coach version which is presented as being more objective and enriching than the assumed blurred, distorted worldview of the naive individual using the app.

By integrating a personal assistant, the applications share the model of human cognitive functioning construed in the heuristics \& biases psychological literature [6], [7]. According to experimental evidence in this field, people in daily situations are poor statisticians: their estimates of risk are widely divergent from normatively correct figures, ignoring baseline values and being mostly dependent on recent incidents (the availability heuristic) and situational cues (anchoring). A related strand of psychology and economics indicates that we value our future selves less than our present selves ('time discounting' or 'hyperbolic discounting' [8]; time discounting is enhanced in cases of addiction [9]). To put it briefly, people in general and smokers in particular are expected: (1) To not know, in quantitative details, specific risks of smoking and 
benefits of quitting; (2) To discount future health benefits in favor of the immediate gratification of nicotine, as a biological agent, and smoking, as habit and social interaction; (3) To ignore health warnings, through various forms of rationalization and exception-based reasoning. The applications, by integrating the advice of a smoking coach, aim to provide users with access to some kind of a privileged knowledge and modes of self-regulation assumed to be superior to the usual, naïve smokers' strategies of cessation.

\section{Burning the Bridge: Moral and Process Identities}

Smoking cessation applications project the user in past, present and future identities. By organizing the process of transformation in different frames, the applications integrates time structures and practices which are prone to establish a dynamic of use and sustain different forms of engagement [17]. Based on temporal arrangements, the applications propose a path of socialization into a new role defined according to users' attachment to cigarettes. Apps propose judgments of right and wrong, worthy and non-worthy, thus defining users' actions as moral or, conversely, immoral. While engaging with the applications users must mitigate between their past identities and the desired ones, in order to become part of the moral order developed through design.

A frequent identity available to users is the future non-smoker. Users are inscribed on a quantified trajectory that connects them with the identity of the non-smoker, as specified through their health risks (Fig. 2a). Quantification is a device that simultaneously brings forward a reality of improved health and finances, and makes possible emotions of enjoyment of one's evolution, and the admiration of the impact of one's actions.

Applications also support the transitional 'smoke-free' identity, dealing with the difficulties of hour-by-hour abstinence. Users are cast as transitional characters, moving from a "risk-laden world" to a "safe haven" of financial and health benefits. This voyage is, though, extremely slippery, and there is no possibility of coming back to a previous estate. Each smoked cigarette reported into the application resets all metrics and brings the user back at square one. In the app world, there is no inertia of benefits: health advantages that have been so vividly materialized, through ever increasing metrics, in previous days, vanish in the smoke of one (reported) cigarette. Gradual accumulation does not entail gradual loss: metrics are used to position users in one or the other of the two worlds, but never in both. Smoking can only occur in the smoking world, from which the travel must start anew. This one-way model of users' trajectory superposes psychological risks with health risks: the psychological damage of relapses, which reaffirm addiction and decrease self-efficacy, is made visible by erasing accumulated health benefits.

Through constant updating, the future becomes incorporated in the present: each day that passes reduces in the here-and-now risks of future disease; this actuality of the future is best captured by what we term 'refreshing quantification', the practice of using precision and updates, in the communication of metrics, as rhetorical resources 
to depict changes in users' bodies. Users receive precise estimates of their health improvement - even if the app, of course, has no individual-level information about users' physical transformation.

Another recurrent moral identity is the Lone Ranger. Most applications promote autonomy as the core value of the smoking cessation project: users are framed as independent, individual fighters against addiction. This identity explains the absence of advice for pharmacotherapy and counseling (with the notable exception of ExSmokers iCoach). The examined applications share a model of their implied users: they are people who have decided to quit smoking and are determined to carry through this radical transformation. The implied users are independent individuals, technologically adept, and honest about their smoking behavior. More importantly, the implied user is the bearer of specific problems that the application purports to solve - but only under the conditions of active involvement. Therefore, moral agency is a key quality of the implied users, which makes possible their interaction with the app.

Local, app-specific identities are also available: the Ultimate Kwitter (Kwit - quit smoking is a game), a quest-driven gamer, or the ExSmoker (ExSmokers iCoach), an 'unstoppable' character, derive their force from the specifics of app use. These identities are achieved by progressing though a scenario in which users establish relationship with objects (digital or real) that might become a substitute for cigarettes.

Through multiple, synchronously evolving quests, the application communicates consistency and intelligibility: saved money, saved time, improved life expectancy. Unlike health risks and benefits, which are experientially distant from the user and come across as interesting scientific but possibly mistrusted 'facts', information about money saved is directly related to the immediate costs of smoking, which the users can observe and influence on their own. Controllable financial and time metrics, derived from reported expenses, compensate for the subjective uncertainty of health benefits that are estimated across large populations, in obvious ignorance of the individual health condition of the user.

Some applications quantify the money saved by each not-smoked cigarette, framing it as a desirable achievement and integrating both past behavior and the anticipation a future reward. This metric corresponds to users' direct experiences of spending money to buy cigarettes. Still, saved money is more abstract than spent money; saved money does not accumulate in a distinctive account, and it may be experienced, ultimately, as a fiction. In order to render this quantitative dimension of the smoke-free world more visible, some applications convert saved money into illustrative goods: "You saved \$5, that's the cost of a Subway Footlong Sandwich", "You saved \$10, that's the cost of a regular movie ticket". "You saved $\$ 50$, that's the cost for a month's membership at the gym". In some applications, such as "Get Rich or Die Smoking", users can assemble personal wishlists of goods to purchase with the money arithmetically saved from smoking, and can share these lists on social media - thus adding an extra-layer of reality derived from others' reactions, a medium to create and share moral significance. 


\section{$5 \quad$ From Broadcast to Dialogue: Rhetorical Devices}

Communication styles range from broadcast (generic messages, such as quitting advice or anti-smoking posters) to interactive, personalized dialogue. Customization is mainly achieved in relation to users' numerical smoking patterns (frequency, cost, duration); tips and advice are, as a rule, not customized to users' preferences or quitting stage.

The style of communication that most of the applications propose is bidirectional: both directed from users to application (when reporting the smoking habits, filing out the surveys, answering questions) and from applications to users (when giving advice, questioning habits, providing statistics or other information). In much complex applications, the communication patterns are based on feedback: users might report whether they are prepared to move on to the next stage or not, whether a tip was useful or not. Also, the apps provide feedback to users confronting a failure or enjoying a success. These possibilities of communication constitute the interactional structure for the mediated relationships and the identities discussed above.

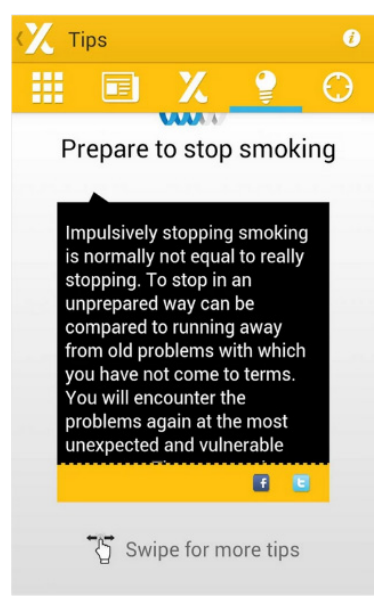

a) 'ExSmoker's ICoach': Tips

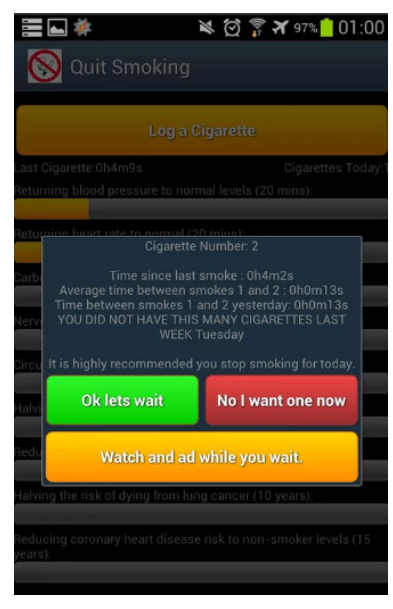

b) 'QuitSmoking': Statistical profile

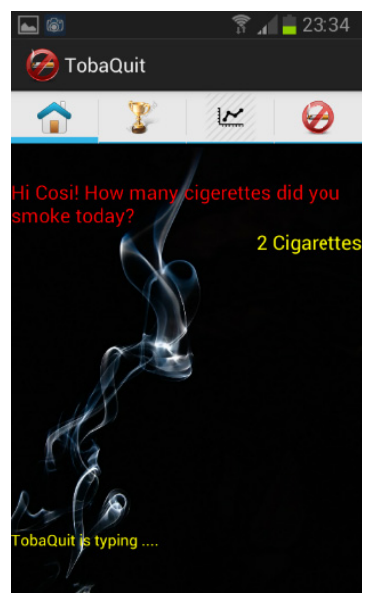

c) 'Tobacco Kills': App anthropomorphism

Fig. 3. Communication and rhetorical strategies

Other modes of personalizing communication include strategies of positioning users within aggregated statistical landscapes computed with information from all other users. Some apps such as 'Quit Smoking: Cessation Nation' provide aggregated and archived data on the situation of the entire community.

Some applications open choice spaces for users (Fig. 3b), mostly designed for situations of craving, when users decide whether or not to smoke a cigarette in that particular moment. Therefore, the question-answer interactions that define the choice are essential not only for their motivational power, but also for the possibility of quantify- 
ing relapses and in ensuring the accurate reset of those achievements and health benefits achievements gained in the period of abstention. There are frequent relapses in the early days of quitting, due to withdrawal difficulties; many smokers quit successfully after several attempts. Several interesting questions arise: For such users, who enjoy the force of 'refreshing quantification' in bringing to life a safe world of gradually diminishing health risks for a couple of days, is this reset understood as a descriptive estimate of risks, or as a punishment for not staying true to one's promise? To what extent does this instability of health benefits undermine the credibility of the metrics, by de-materializing and conventionalizing the numbers? How do users who relapse communicate with the application in this choice space? Do they report honestly, or do they omit the occasional cigarette so that they would benefit from the motivational force of seeing the safe haven of a smoke free life? These questions invite further empirical scrutiny, in relation to evidence of high relapse rates [10], [11].

Other rhetorical tools of dialogue include both using one's first name (surprisingly rare, although preferred by users [18]) or apps that impersonate a human: "TobaQuit is typing" (Fig. 3c). Through forms of direct address, probabilities (reduced risks, increased benefits) become facts about oneself. Probabilities are freed from the doubt of being past approximations of other people's fates, and are rendered into accurate, actual description of transformations in one's own body and life.

Rhetorical strategies also include advice tips that are intimate (concerning one's sensibilities), surprising, or humoristic, thus eliciting emotions. For example, applications address in detail personal feelings: "Be prepared for a rollercoaster ride of emotions: denial, anger, bargaining, depression, acceptance, and complacency. These are normal emotions for your body when going through withdrawal" (Stop Smoking Assistant) or "Everything has its time, even your panic. Instead of being afraid of it: observe the feeling, it is something you can bear" (Exsmokers iCoach). Through these tips, apps not only anticipate future feelings or help users introspectively recognize their actual states, but, by focusing attention and by labeling such emotions, they create the experiences that they describe. This is yet another way in which apps actively shape users' experiences of quitting smoking.

\section{Conclusions}

Mobile applications for smoking cessation are a growing form of support for people who attempt to quit the habit. By an empirical analysis of Android OS applications, we observe their communication patterns and the projects through which they purport to assist learners.

In order to center users' identities on the core values of freedom and autonomy, mobile apps propose a temporal order in which the past (quantification of previous behavior), present (craving management tools) and future (personalized predictions) are addressed concomitantly. Users are encouraged to shift from one temporal realm into another in order to improve their ability to face obstacles when desiring or craving to smoke. Apps also claim to give users access to scientifically-formulated, privileged knowledge about smoking risks and cessation benefits. By transmitting this 
information and by a strong moral framing of smoking, apps render users morally accountable for their actions.

We observe several ways in which the experience of smoking cessation is coconstructed in interaction between users, application and peers. Apps provide users with clues of interpreting their past, present and future behavior. Beside this, they often rely on 'refreshing quantification' to construct a shared language and a shared worldview with their users, in which numbers bring to the touchscreen a better, healthier life.

Many applications frame smoking cessation as a heroic, individual pursuit: smokers are invited, without any equipment but their mobile devices, to enhance their probabilistic vision in order to carry through a lonely, one-way travel from danger to safety. Relapse is punished by return to square one: health benefits are thus moralized, functioning less as a quantitative description of one's body than as an indicator for the moral statuses of 'smoker' or 'former smoker'. Given the high prevalence of relapse, especially in early days, it is important to investigate empirically how people relate to this implied user model, and whether it could be improved.

Acknowledgments. This article has been supported by the research project "Sociological imagination and disciplinary orientation in applied social research", with financial support of ANCS / UEFISCDI grant no. PN-II-RU-TE-2011-3-0143, contract no. $14 / 28.10 .2011$.

\section{References}

1. Civljak, M., Stead, L., Hartmann-Boyce, J., Sheikh, A., Car, J.: Internet-based interventions for smoking cessation. The Cochrane Database of Systematic Reviews 7 (2013)

2. Whittaker, R., McRobbie, H., Bullen, C., Borland, R., Rodgers, A., Gu, Y.: Mobile phonebased interventions for smoking cessation. The Cochrane Database of Systematic Reviews 11, 1-24 (2012)

3. Abroms, L., Padmanabhan, N., Thaweethai, L., Phillips, T.: iPhone Apps for Smoking Cessation: A Content Analysis. American Journal of Preventive Medicine 40(3), 279-285 (2011)

4. Sharrock, W., Anderson, B.: The user as a scenic feature of the design space. Design Study 15(1), 5-18 (1994)

5. Mads, B., Brown, N., Konrad, K., Harro Van, L.: The Sociology of Expectations in Science and Technology. Technology Analysis \& Strategic Management 18(3-4), 285-298 (2006)

6. Johansson, M., Messeter, J.: Presenting the user: constructing the persona. Digital Creativity 16(4), 231-243 (2005)

7. Oudshoorn, N., Rommes, E., Marcelle, S.: Configuring the User as Everybody: Gender and Design Cultures in Information and Communication Technologies. Science, Technology, \& Human Values 29(1), 30-63 (2004)

8. Rooksby, J., Martin, D., Rouncefield, M.: Users as contextual features of software product development and testing. In: Group 2007, pp. 4-7 (2007)

9. Woolgar, S.: Configuring the user: the case of usability trials. In: Law, J. (ed.) A Sociology of Monsters: Essays on Power, Technology and Domination, pp. 57-102 (1991) 
10. Higgins, V.: Performing Users: The Case of a Computer-Based Dairy Decision-Support System. Science, Technology \& Human Values 32, 263-286 (2007)

11. Möller, A., Scherr, J., Roalter, L., Diewald, S., Hammerla, N., Plötz, T., Olivier, P., Kranz, M.: GymSkill: Mobile Exercise Skill Assessment to Support Personal Health and Fitness. In: Adjunct Proceedings of 9th International Conference on Pervasive Computing Pervasive (2011)

12. Brandt, C., Dalum, P., Thomsen, T.: 'I miss the care even though I know it's just a machine': An explorative study of the relationship between an Internet-based smoking cessation intervention and its participants. Health Informatics Journal 19(3), 233-243 (2013)

13. Rughini, R.: Talkative Objects in Need of Interpretation. Re-Thinking Digital Badges in Education. In: AltCHI 2013, ACM SIGCHI Conference on Human Factors in Computing Systems, pp. 1-10 (2013)

14. Rughiniş, R., Matei, S.: Digital Badges: Signposts and Claims of Achievement. In: Stephanidis, C. (ed.) Posters, Part II, HCII 2013. CCIS, vol. 374, pp. 84-88. Springer, Heidelberg (2013)

15. Rughini, R.: Badge Architectures in Engineering Education. Blueprints and Challenges. In: The 5th International Conference on Computer Supported Education, CSEDU 2013 (2013)

16. Rughini, R.: Gamification for Productive Interaction. Reading and Working with the Gamification Debate in Education. In: The 8th Iberian Conference on Information Systems and Technologies, CISTI 2013, pp. 1-5 (2013)

17. Rughini, R.: Time as a Heuristic in Serious Games for Engineering Education. In: The 5th International Conference on Computer Supported Education CSEDU (2013)

18. Jamison, J., Naughton, F., Gilbert, H., Sutton, S.: Delivering Smoking Cessation Support by Mobile Phone Text Message: What Information do Smokers Want? A Focus Group Study. Journal of Applied Biobehavioral Research 18(1), 1-23 (2013) 